\title{
Parasitics Extraction Involving 3-D Conductors Based on Multi-layered Green's Function
}

\author{
Zuochang Ye, Zhiping Yu \\ Institute of Microelectronics, Tsinghua University, Beijing, 100084, China \\ yzc02@mails.tsinghua.edu.cn
}

\begin{abstract}
An efficient algorithm for three-dimensional (3D) capacitance extraction on multi-layered and lossy substrate is presented. The new algorithm represents a major improvement over the quasi-3D approach used in Green's function-based solvers by taking into consideration of the side-wall effects of the conductors. The accuracy and efficiency of the new algorithm is tested by examples.
\end{abstract}

\section{INTRODUCTION}

It has become more critical that 3-D effects should be considered accurately in modeling capacitances on RF CMOS chips because of shrunk feature size and increasing operation frequency. Boundary element method (BEM) is an efficient method for this problem, since only surfaces and interfaces between regions need to be discretized, which greatly reduced the problem compared to volume-discretized methods such as finite difference method (FDM) and finite element method (FEM), e.g., Raphael [1] and HFSS [2].

Great efforts have been made in the BEM-based 3-D capacitance extraction, such as fast multi-pole (FMM) [3], singular value decomposition (SVD) [4], hierarchical [5], and quasimultiple medium (QMM) [6] methods . All these methods, however, mainly focus on the fast solving of the linear system. In this paper, we study the computation of potential coefficient matrix $\mathbf{P}$ (to be defined later) for multi-layered and lossy substrate based on Green's function [7, 8]. In this method, the Green's function is calculated and stored based on the process technology only, thus the dielectric interfaces don't need to be discretized. As a result, the size of the linear system is greatly reduced.

In [7], the chip structure is assumed to be confined by rectangle electric and magnetic walls. Considering the Neumann boundary condition at the magnetic walls, the Green's function can be expanded to cosine series, such that fast Fourier transform (FFT) can be applied to accelerate the calculation of cosine series summation. By introducing the concept of complex permittivity [9], this method can handle lossy substrate easily with little extra efforts. The original algorithm was improved in [8] and implemented in software ASITIC [10].

In ASITIC, however, since only analytical integration over horizontal panels is derived, the conductors are restricted to two dimensional sheets. To extend the capacitance extraction to 3-D domain, the Green's function should be integrated analytically in $z$-direction. However, this meets some difficulty. Because in [8], to preserve the numerical stability, the Green's function in $z$-direction is obtained through a complicated recursive procedure. The resulted expression is a continual multiplication, which cannot be integrated analytically. Although measures are taken in [11] by considering the bottom and top plates of conductors, the sidewall capacitance still cannot be handled. In [12], the conductors are cut horizontally into slices in order to include 3-D effects, but this amounts to volume discretization, leading to prohibitive extra cost.

In this paper, we use a new formula for recursive computation of the Green's function, such that it can be integrated stably in $z$-direction. The formula for the computation of the potential coefficients involving sidewall integration is derived to accomplish the true 3-D parasitics extraction.

\section{GREEN's FunCtion APPROACH to CAPACITANCE EXTRACTION WITH LOSSY SUBSTRATE}

The substrate is characterized in [7] as a multi-layered structure as shown in Fig. 1. Each layer has a thickness and a uniform permittivity and conductivity. Conductors are embedded in the layers. The objective of the parasitics extraction is to compute the $m \times m$ capacitance matrix $\mathbf{C}$ for an $m$-conductor geometry.

To determine the $j$-th column of $\mathbf{C}$, we need only to solve for the surface charges on each conductor produced by raising conductor $j$ to unit potential while grounding rest of the conductors. Then $C_{i j}$ is numerically equal to the charge $q_{i}$ on conductor $i$. This procedure is repeated $m$ times to compute all columns of $\mathbf{C}$. The charge $q_{i}$ is obtained by solving the linear system

$$
\mathbf{P q}=\mathbf{v}
$$

where $\mathbf{v}$ is the given potential vector and $\mathbf{P}$ is the so-called potential coefficient matrix. Each entry of $\mathbf{P}$, i.e. $p_{i j}$, is computed by convolving the charge distribution with the Green's function as

$$
p_{i j}=\frac{1}{A_{i} A_{j}} \int_{\mathbf{r}^{\prime} \in A_{i}} \int_{\mathbf{r} \in A_{j}} G\left(\mathbf{r}^{\prime}, \mathbf{r}\right) d \mathbf{r} d \mathbf{r}^{\prime},
$$


where $A_{1}$ and $A_{2}$ are the areas of the two panels. The Green's function $G\left(\mathbf{r}^{\prime}, \mathbf{r}\right)$ can be computed by solving the Laplace equation

$$
\nabla G\left(\mathbf{r}^{\prime}, \mathbf{r}\right)=\frac{-\delta_{m}\left(\mathbf{r}-\mathbf{r}^{\prime}\right)}{\dot{\varepsilon}_{k}}
$$

where

$$
\dot{\varepsilon}=\varepsilon+\frac{\sigma}{j \omega}
$$

is the complex medium permittivity [9]. By the use of $\dot{\varepsilon}$ instead of $\varepsilon$, both ohmic and displacement currents are accounted for, thus the frequency-dependent effect for conductive substrate is included.

The Greens function for the boundary condition shown in Fig. 1 can be solved as a double infinite summation

$$
\begin{array}{r}
G\left(x^{\prime}, y^{\prime}, z^{\prime}, x, y, z\right)=\sum_{m=0}^{\infty} \sum_{n=0}^{\infty} f_{m n}\left(z^{\prime}, z\right) \times \\
\cos \left(\delta_{m} x^{\prime}\right) \cos \left(\xi_{n} y^{\prime}\right) \cos \left(\delta_{m} x\right) \cos \left(\xi_{n} y\right),
\end{array}
$$

where $\delta_{m}=m \pi / a, \xi_{n}=n \pi / b, a, b$ are the substrate lateral dimensions. $f_{m n}$ is obtained by solving the Laplace equations in the $z$-direction along with the boundary conditions in this dimension. In [7], $f_{m n}$ is composed of hyperbolic functions, while in this paper, it is expressed in an exponential manner as

$$
\begin{aligned}
f_{m n}\left(z^{\prime}, z\right)=\frac{C_{m n}}{a b \dot{\varepsilon}_{s} \gamma} \times \\
\frac{\left(\alpha_{s}^{u, l} e^{\gamma z^{\prime}}+\beta_{s}^{u, l} e^{-\gamma z^{\prime}}\right)\left(\alpha_{f}^{l, u} e^{\gamma z}+\beta_{f}^{l, u} e^{-\gamma z}\right)}{\alpha_{s}^{u} \beta_{s}^{l}-\alpha_{s}^{l} \beta_{s}^{u}} \\
=\left(\alpha_{s} e^{\gamma z^{\prime}}+\beta_{s} e^{-\gamma z^{\prime}}\right)\left(\alpha_{f} e^{\gamma z^{\prime}}+\beta_{f} e^{-\gamma z^{\prime}}\right),
\end{aligned}
$$

where $C_{m n}=4$ for $(m, n>0), C_{m n}=2$ for $(m=0$ or $n=0$ but not both being zero), $C_{00}=1$ and $\gamma=$ $\sqrt{(m \pi / a)^{2}+(n \pi / b)^{2}}$. The subscript ' $s$ ' denotes the source layer, and the subscript ' $f$ ' denotes the field layer. The coefficients $\alpha_{s}^{u, l}, \alpha_{f}^{u, l}, \beta_{s}^{u, l}, \beta_{f}^{u, l}$ can be derived with the help of recursive formula, similar to the one in [7].

The major advantage of using the exponential function to express $f_{m n}$ is that it makes it feasible to integrate $f_{m n}$ in $z$ direction analytically and stably, which cannot be done using the expression in [8]. In the next section, the computation involving integration in $z$-direction will be presented in detail.

\section{DERIVATION OF THE PANEL INTEGRATIONS FOR 3-D EXTRACTION}

Suppose the coordinates for two conductors are as shown in Fig. 1. Each of the conductors' faces is treated as an independent panel. To find $p_{i j},(5)$ is integrated over the surfaces of panel $i$ and panel $j$. If one substitutes (5) in (2), and interchanges the order of the operations, the integral can be computed analytically, leaving a 2-D infinite summation.
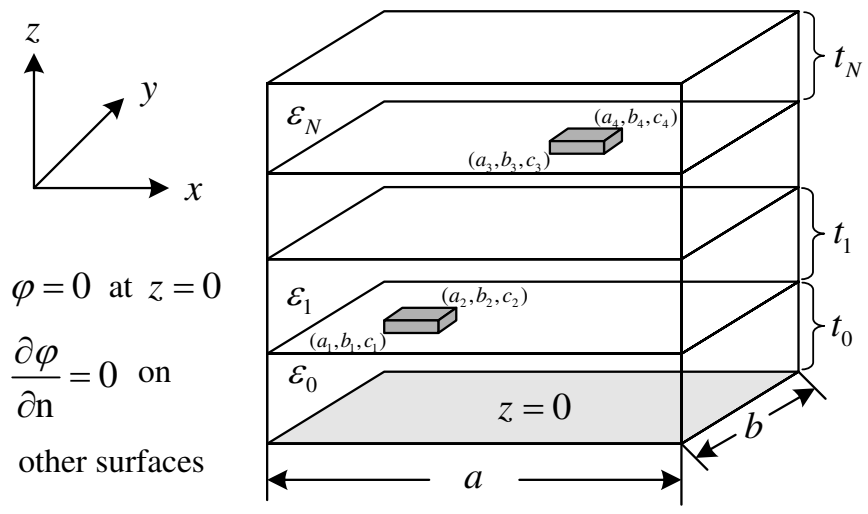

Fig. 1. Geometry and boundary condition of multi-layer substrate

To compute the 3-D capacitance, the panels of a cuboid conductor is grouped into three classes, horizontal (in $x$ - $y$ plane), vertical I (in $x-z$ plane) and vertical II (in $y-z$ plane). The positional combination of panel pairs can be classified as four types as shown in Fig. 2. In type A, the two panels are both horizontal ones, i.e., on the bottom or the top surfaces of the conductors. In type B, one of the panels is horizontal, and the other is vertical. In types $\mathrm{C}$ and $\mathrm{D}$, both panels are vertical. They are distinguished by whether the panels are parallel or perpendicular to each other. In the following subsections, each type of panel pairs is discussed in turn.

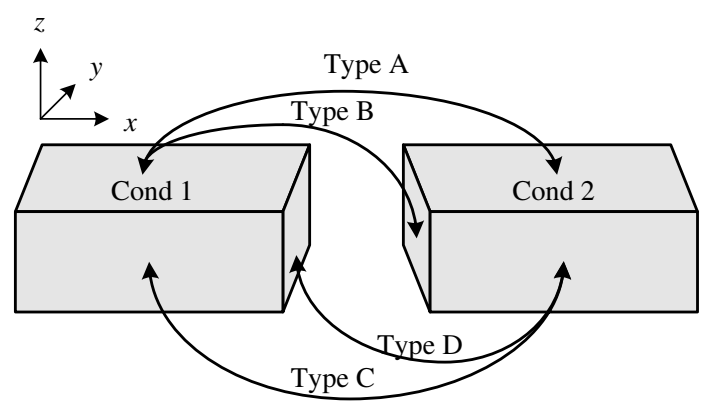

Fig. 2. Positional relations of two panels

\section{A. Horizontal-Horizontal (Type A)}

To compute the potential coefficient of two panels in $x-y$ plane, the Green's function is integrated over them. Excluding the $m=0$ and $n=0$ case, it can be written as [8]

$$
\begin{gathered}
p_{i j}=\sum_{m=1}^{S-1} \sum_{n=1}^{S-1} \frac{f_{m n}\left(z, z^{\prime}\right)}{A_{1} A_{2}} \int_{a_{1}}^{a_{2}} \int_{b_{1}}^{b_{2}} \int_{a_{3}}^{a_{4}} \int_{b_{3}}^{b_{4}} \times \\
\cos \left(\delta_{m} x^{\prime}\right) \cos \left(\xi_{n} y^{\prime}\right) \cos \left(\delta_{m} x\right) \cos \left(\xi_{n} y\right) d y d x d y^{\prime} d x^{\prime},
\end{gathered}
$$

where $S$ is the upper bound of the truncated infinite series. The integration in (7) can be performed easily, resulting in sine 
functions, and it can be cast into a sum of 64 terms in the form

$$
p_{i j}=\sum_{m=1}^{S-1} \sum_{n=1}^{S-1} \frac{f_{m n}}{A_{1} A_{2} \delta_{m}^{2} \xi_{n}^{2}} \cos \left[\delta_{m}\left(a_{1,2} \pm a_{3,4}\right)\right] \cos \left[\xi_{n}\left(b_{1,2} \pm b_{3,4}\right)\right]
$$

Thus, for a specific technology, the matrix of DCT (discrete cosine transform), i.e.

$$
K_{p q}=\sum_{m=1}^{S-1} \sum_{n=1}^{S-1} \frac{f_{m n}}{\delta_{m}^{2} \xi_{n}^{2}} \cos \left(m \pi \frac{p}{S}\right) \cos \left(n \pi \frac{q}{S}\right),
$$

can be computed beforehand and stored in a database for $1 \leq$ $p, q \leq S$. When the layout information, i.e. the coordinates $a_{1-}$ $a_{4}, b_{1}-b_{4}$, is given, the potential coefficients can be obtained by simply access in matrix $\mathbf{K}$ and summating the 64 terms.

The computation of (9) need to be computed only once for a given technology, and it can be very efficient by use of the fast Fourier transform. For more detail refer to [7].

\section{B. Horizontal-Vertical (Type B)}

For the case of type B, since a vertical panel is involved, the computation of potential coefficients consists of three integrations in $x$ - and $y$-directions and one integration in $z$-direction. With the presence of (6), it is very easy to perform the analytical integration of $f_{m n}\left(z^{\prime}, z\right)$ in $z$-direction:

$$
f_{m n}^{(1)}=\int_{c_{1}}^{c_{2}} f_{m n}\left(z^{\prime}, z\right) d z
$$

The remaining three integrations in $x-y$ plane are the same as in type A. Suppose the vertical panel is field panel, and it is in $x-z$ plane with $y$-coordinate being $b_{f}$, the integration of potential coefficient can be written as

$$
\begin{aligned}
& p_{i j}= \sum_{m=1}^{S-1} \sum_{n=1}^{S-1} \frac{\cos \left(\xi_{n} b_{f}\right)}{A_{1} A_{2}} \int_{a_{1}}^{a_{2}} \int_{b_{1}}^{b_{2}} \int_{a_{3}}^{a_{4}} \int_{c_{3}}^{c_{4}} \times \\
& \cos \left(\delta_{m} x^{\prime}\right) \cos \left(\xi_{n} y^{\prime}\right) \cos \left(\delta_{m} x\right) f_{m n}\left(z, z^{\prime}\right) d z^{\prime} d x d y^{\prime} d x^{\prime} \\
&=\sum_{m=1}^{S-1} \sum_{n=1}^{S-1} \frac{f_{m n}^{(1)}}{A_{1} A_{2} \delta_{m}^{2} \xi_{n}}\left[\sin \left(\xi_{n} b_{2}\right)-\sin \left(\xi_{n} b_{1}\right)\right] \cos \left(\xi_{n} b_{f}\right) \times \\
& \quad\left[\sin \left(\delta_{m} a_{2}\right)-\sin \left(\delta_{m} a_{1}\right)\right]\left[\sin \left(\delta_{m} a_{4}\right)-\sin \left(\delta_{m} a_{3}\right)\right] .
\end{aligned}
$$

It can be cast into a sum of 32 terms just like in the case of type A, and it can be recast into the form of discrete cosinesine transform (DCST)

$$
K_{p q}=\sum_{m=1}^{S-1} \sum_{n=1}^{S-1} \frac{f_{m n}^{(1)}}{\delta_{m}^{2} \xi_{n}} \cos \left(m \pi \frac{p}{S}\right) \sin \left(n \pi \frac{q}{S}\right)
$$

which is very similar to (9), and it can also be computed rapidly by the use of the fast Fourier transform.

\section{Vertical-Vertical (Types $C, D)$}

For types $\mathrm{C}$ and $\mathrm{D}$, the two panels are both vertical. The computation of $p_{i j}$ consists of two integrations in $x-y$ plane and two integrations in $z$-direction. Since the coefficients are discontinuous at the source point, depending on whether the two panels are in the same layer or not, the analytical integration in $z$-direction

$$
f_{m n}^{(2)}=\int_{c_{1}}^{c_{2}} \int_{c_{3}}^{c_{4}} f_{m n}\left(z^{\prime}, z\right) d z d z^{\prime}
$$

should be derived individually, and it can be done with the help of mathematical tools, such as Mathematica [13].

The remaining derivation of $p_{i j}$ includes two integrations in $x-y$ plane, and the resulted matrix $\mathbf{K}$ are DCT (for type C) and DST (discrete sine transform, for type D), respectively. Due to length limit, the detail will not be presented here.

\section{NUMERICAL RESULTS}

The algorithm described above is implemented in a solver named SCAPE (Substrate Coupling Analyzer for Passive Elements). In this section, several examples will be shown to verify SCAPE and to compare the accuracy and efficiency with other methods. The simulations are done on a Sun Blade 2000 with Ultra SPARC III Cu processors at $900 \mathrm{MHz}$ and $2 \mathrm{~GB}$ memory.

The test cases are $k \times k(k=2 \sim 5)$ crossing conductors in five dielectrics as shown in Fig. 3. The size of each conductor is $(2 k+1) \times 1 \times 1$ (unit in micron). The spacing between neighboring conductors in the same layer is $1 \mu \mathrm{m}$. The relative permittivity of every dielectric layer is 3.9 . The thickness is 28 for the top layer and 1 for the rest layers. Each of the structures are surrounded by a $32 \times 32 \times 32$ box. The bottom of the box is a perfect ground plane, and the other five surfaces of the box are Neumann boundaries.

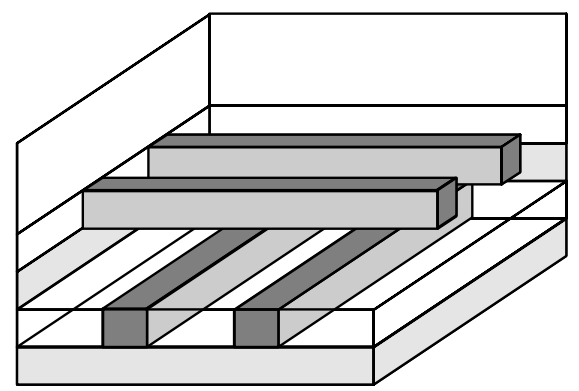

Fig. $3.2 \times 2$ crossover in dielectrics

The above crossover problems are computed by FastCap with very fine meshing, denoted by FastCap I, FastCap with relative coarse meshing, denoted by FastCap II, ASITIC, and SCAPE. Both the expansion order of FastCap I and FastCap II is 2. It is the most accurate version of FastCap, within a 
reasonable amount of time. In the input of FastCap, 0 is assigned to the permittivity of outer space to handle the boundary condition, and each interface between the dielectric layers is specified to make the comparisons equitable.

In ASITIC, since it can not handle conductors with finite thickness, to model thickness effect to the best of its abilities, the upper and lower surfaces of conductors are treated independently as conductors of zero thickness and extracted as such [11]. After extraction, the resulted capacitance matrix is reduced by combining together the top and the bottom plates of the conductors. This is electrically equivalent to shorting the top and the bottom plates together.

Using the capacitance matrix $\mathbf{C}$ computed by FastCap I, i.e. FastCap with very fine meshing, as the standard, the error of the capacitance matrix $\mathbf{C}^{\prime}$ computed by another program is estimated in the two-norm: $\left\|\mathbf{C}^{\prime}-\mathbf{C}\right\| /\|\mathbf{C}\|$. The error of FastCap II is set to around $3 \%$, controlled by adjusting the mesh size.

Table I compares SCAPE with ASITIC and FastCap. The following is a summary of the comparison.

1. Using FastCap I's result as criterion, SCAPE is more accurate than FastCap II, which is within $2.5 \%$ and around 3\%, respectively. ASITIC does not account for the sidewall effect, so its error is unacceptably large (>20\%).

2. Since SCAPE does not need to discretize the ground plane, the boundary, and the dielectric interfaces, it uses much fewer panels than FastCap I and II, thus it is much faster than them. The speedup compared to FastCap I is 169 to 42, and 36 to 6 to FastCap II.

3. SCAPE uses $1 / 14$ to $1 / 6$ of the memory used by FastCap I, and about $1 / 3$ of the memory used by FastCap II. The memory required by SCAPE grows slower than FastCap does with the problem size grows. This is because in SCAPE most of the memories are used to store the Green's function, which is fixed when the problem size grows. So it is better for large scale problems.

It is worth noting that, in the above simulations with FastCap, the substrate is only $32 \mu \mathrm{m} \times 32 \mu \mathrm{m}$, which is much smaller than reality. For an actual technology, the problem size for FastCap will increase drastically, while for the new algorithm it is fixed, since the dielectric interface charge is accounted for during the computation of Green's function.

\section{CONCLUSION}

The multi-layered Green's function-based algorithm proposed in [7] for substrate coupling analysis is extended to 3$\mathrm{D}$ domain. To accomplish this, a new recursive formula for computing the Green's function is derived and it is integrated analytically over the sidewall surfaces. Test examples show that it brings prominent improvement in accuracy, compared to ASITIC.
TABLE I

COMPARISON FOR $k \times k$ BUS PROBLEMS.

\begin{tabular}{|c|c|c|c|c|}
\hline \multicolumn{5}{|c|}{ Test problem } \\
\hline & $2 \times 2$ & $3 \times 3$ & $4 \times 4$ & $5 \times 5$ \\
\hline \multicolumn{5}{|c|}{ FastCap I (with fine mesh) } \\
\hline CPU Time (s) & 54 & 120 & 218 & 349 \\
\hline Memory (MB) & 111 & 131 & 160 & 197 \\
\hline Panel \# & 7812 & 8724 & 9948 & 11492 \\
\hline \multicolumn{5}{|c|}{ FastCap II (with coarse mesh) } \\
\hline CPU Time (s) & 11.6 & 19.1 & 31.1 & 46.6 \\
\hline Memory (MB) & 56 & 64 & 75 & 89 \\
\hline Panel \# & 3628 & 4080 & 4684 & 5448 \\
\hline Error $(\%)$ & 2.89 & 2.84 & 2.85 & 2.76 \\
\hline \multicolumn{5}{|c|}{ ASITIC } \\
\hline Panel \# & 50 & 84 & 144 & 220 \\
\hline Error $(\%)$ & 23.7 & 24.8 & 25.8 & 25.4 \\
\hline \multicolumn{5}{|c|}{ SCAPE } \\
\hline CPU time (s) & 0.32 & 1.14 & 3.41 & 8.39 \\
\hline Memory (MB) & 19 & 20 & 21 & 25 \\
\hline Panel \# & 146 & 276 & 464 & 700 \\
\hline Error $(\%)$ & 0.99 & 0.91 & 1.60 & 2.38 \\
\hline
\end{tabular}

\section{ACKNOWLEDGMENTS}

This work is supported by a research grant (2004AA1Z11050) from the National 863 Plan sponsored by the Ministry of Science and Technology in China. The collaboration with Cadence in San Jose, U.S. is greatly appreciated.

\section{REFERENCES}

[1] Raphael User's Manual, Synopsys, 2003.

[2] HFSS User Manual, Ansoft, 2002.

[3] K. Nabors and J. White, "FastCap: a multipole accelerated 3-d capacitance extraction program," IEEE Trans. Computer-Aided Design, vol. 10, no. 11, pp. 1447-1459, Nov. 1991.

[4] S. Kapur and D. E. Long, "IES3 : A fast integral equation solver for efficient 3-dimensional extraction," in Proceedings of International Conference on Computer Aided Design, San Jose, CA, USA, Nov. 1997, pp. 448-455.

[5] W. Shi, J. Liu, N. Kakani, and T. Yu, "A fast hierarchical algorithm for three-dimensional capacitance extraction," IEEE Trans. Computer-Aided Design, vol. 21, no. 3, pp. 330-336, Mar. 2002.

[6] W. Yu and Z. Wang, "Enhanced QMM-BEM solver for three-dimensional multiple-dielectric capacitance extraction within the finite domain," IEEE Trans. Microwave Theory Tech., vol. 52, no. 2, pp. 560-566, Feb. 2004. 
[7] R. Gharpurey and R. G. Meyer, "Modeling and analysis of substrate coupling in integrated circuits," IEEE J. Solid-State Circuits, vol. 31, no. 3, pp. 344-353, Mar. 1996.

[8] A. M. Niknejad, R. Gharpurey, and R. G. Meyer, "Numerically stable Green function for modeling and analysis of substrate coupling in integrated circuits," IEEE Trans. Computer-Aided Design, vol. 17, no. 4, pp. 305315, Apr. 1998.

[9] S. Ramo, J. R. Whinnery, and T. V. Duzer, Fields and Waves in Communication Electronics, 3rd ed. New York: Wiley, 1994.

[10] A. M. Niknejad and R. G. Meyer, "Analysis, design, and optimization of spiral inductors and transformers for $\mathrm{Si}$
RF IC's," IEEE J. Solid-State Circuits, vol. 33, no. 10, pp. 1470-1481, Oct. 1998.

[11] R. Gharpurey and S. Hosur, "Transform domain techniques for efficient extraction of substrate," in Proceedings of International Conference on Computer Aided Design, San Jose, California, 1997, pp. 461-467.

[12] R. Gharpurey, "Modeling and Analysis of Substrate Coupling in Integrated Circuits," Ph.D. dissertation, Collage of Engineering, University of California at Berkeley, 1999.

[13] Mathematica User's Guide, Wolfram Research, 2003. 